\title{
Gastric linitis plastica: which role for surgical resection?
}

\author{
Corrado Pedrazzani $\cdot$ Daniele Marrelli $\cdot$ Fabio Pacelli $\cdot$ \\ Maria Di Cosmo • Gianni Mura • Francesca Bettarini • \\ Fausto Rosa • Giovanni de Manzoni · Franco Roviello
}

Received: 29 September 2010/Accepted: 17 May 2011/Published online: 30 June 2011

(c) The International Gastric Cancer Association and The Japanese Gastric Cancer Association 2011

\begin{abstract}
Background The role of surgery for gastric linitis plastica (GLP) is questioned. This study aimed to analyze our experience in the surgical treatment of GLP with specific reference to the resectability rate, prognosis, and mode of recurrence.

Methods Results of surgery were analyzed in 102 patients with GLP.

Results Of the 102 patients, 92 underwent surgical exploration, with resection performed in 60 cases. R2 resection was carried out in 20 patients and R1 in 12 patients, while the resection was considered potentially curative (R0) in 28 (27.5\%). Overall, the median $(95 \%$
\end{abstract}

On behalf of the Italian Research Group for Gastric Cancer Study.

C. Pedrazzani - D. Marrelli · F. Bettarini · F. Roviello

Unit of Surgical Oncology, Department of Human Pathology and Oncology, Istituto Toscano Tumouri, University of Siena and ITT, Siena, Italy

F. Pacelli · F. Rosa

Department of Digestive Surgery,

Catholic University of Rome, Rome, Italy

M. Di Cosmo - G. de Manzoni

Department of General Surgery,

University of Verona, Verona, Italy

G. Mura

Division of General Surgery,

Valdarno Hospital, Valdarno, Arezzo, Italy

C. Pedrazzani $(\bowtie)$

Sezione di Chirurgia Oncologica,

Dipartimento di Patologia Umana e Oncologia,

Policlinico 'Le Scotte', 53100 Siena, Italy

e-mail: pedrazzani@unisi.it confidence interval [CI]) survival time was 5.7 (3.7-7.5) months, with none of the patients alive at the end date of the study. For R0 patients the median $(95 \% \mathrm{CI})$ survival time was 15.8 (11-20.7) months. The great majority of recurrences were intra-abdominal (peritoneal and/or locoregional), with a systemic component of the relapse that was rarely observed (5 cases).

Conclusions After primary surgery, GLP showed a poor prognosis without regard to the extent or type of resection. The failure of surgical treatment related mainly to the peritoneal spread of the disease. Specifically designed multimodality treatment protocols should be tested in this setting.

Keywords Gastric cancer - Linitis plastica - Surgery · Prognosis

\section{Introduction}

Gastric cancer (GC) is one of the leading causes of cancerrelated death worldwide. The incidence of intestinal-type GC is decreasing in Western countries, although the number of diffuse-type cancers remains stable [1]. No specific epidemiological data are available for gastric linitis plastica (GLP), although this type of tumor is stably observed in clinical practice, accounting for $3-19 \%$ of GC cases [2].

Although the prognosis of GLP is commonly considered poor, the number of reports addressing this topic is quite small.

The purpose of this study was to analyze our experience in the surgical treatment of GLP with specific reference to the resectability rate, overall survival, and mode of recurrence. 


\section{Methods}

\section{Definition}

GLP was defined as a GC of the diffuse histotype according to Lauren [3] that by diffuse infiltration caused thickening and stiffening of the gastric wall and involved circumferentially at least one-third of the stomach. GLP frequently involved the stomach from the fundus to the pylorus.

Population under study

This study analyzed 102 patients who were evaluated from January 1990 to December 2007 in four Italian surgical departments (Department of General Surgery and Surgical Oncology, University of Siena; First Department of General Surgery, University of Verona; Department of Digestive Surgery, Catholic University of Rome; Division of General Surgery, Valdarno, Arezzo) that are part of the Italian Research Group for Gastric Cancer (IRGGC).

The study was approved by the local ethics committees.

\section{Staging work-up}

Clinical staging included esophagogastroduodenoscopy (EGD) with biopsy barium swallow, and thoracoabdominal computed tomography (CT) in every case. Endoscopic ultrasonography (EUS) and other investigations were performed according to physician's judgments. Staging laparoscopy was never employed during the time of the study.

Extent of gastric resection and lymphadenectomy

The main goal of surgery was to achieve the complete removal of the tumor ( $\mathrm{R} 0$ resection), although palliative procedures were carried out in order to treat tumor-related complications.

Total gastrectomy was usually preferred, although distal subtotal gastrectomy was performed in selected cases; namely, in tumors located in the lower third of the stomach provided that the proximal resection margin stayed at least $6 \mathrm{~cm}$ from the tumor edge (9 cases).

In potentially curative gastrectomy, an extended lymph node dissection is routinely carried out at the four institutions; likewise, at the four institutions, D2-3 lymphadenectomy was employed in fit patients. Lymphadenectomy was classified and standardized in accordance with the Japanese Gastric Cancer Association (JGCA) guidelines [4]. The median (range) number of excised nodes was 36 (15-88). Lymphadenectomy at the splenic hilum was performed in patients with tumors reaching the greater curvature and the gastric fundus or with enlarged nodes at the splenic hilum. This was achieved by performing a pancreas-preserving splenectomy (11 cases).

No patient in the cohort was proposed to undergo preoperative chemotherapy and/or radiotherapy.

Staging and lymph node classification

Tumors were staged according to the pathological classification (pTNM) of the American Joint Committee on Cancer (AJCC) [5]. Lymph node involvement was classified according to the number (AJCC) [5] and site (JGCA) [4] of nodal metastasis.

Follow-up and data analysis

All patients included in the study were regularly followedup with a standardized protocol [6]. The type of recurrence was classified as follows: (1) locoregional if tumor was detected in the surgical anastomosis, gastric bed, or abdominal lymph nodes; (2) peritoneal if tumor recurred as peritoneal carcinosis; (3) hematogenous if distant metastases were observed (liver, lung, bone, or lymph nodes outside the abdomen). Only the first site of relapse was taken into consideration. Any metastatic involvement observed after identification of the first recurrence was not considered. Survival curves were estimated using the Kaplan-Meier method and compared by the Log-rank test, with the time of survival measured from the date of hospital admission to the date of death. Analyses were performed using the Statistical Package for the Social Sciences, SPSS 16.0 for Windows, XP (SPSS, Chicago IL, USA).

\section{Results}

Clinico-pathological characteristics

Of the 102 patients with a median (range) age of 68 (29-89) years, a total of 45 were females. Table 1 reports the main demographic and clinical characteristics of the cohort under study.

\section{Resection rate}

After completion of the staging work-up, 92 patients underwent surgical exploration, while 10 patients did not. At the time of laparotomy, the tumor was judged nonresectable in 32 patients, whereas resective surgery was performed in 60. A palliative (R2) resection was carried out in 20 patients, microscopic residual (R1) tumor was observed in 12 (positive peritoneal washing in 9 cases and microscopic involvement of proximal resection margin in 
Table 1 Main clinico-pathological characteristics and survival data of the 102 patients under study

\begin{tabular}{|c|c|c|c|}
\hline Variable & $\begin{array}{l}\text { No. of } \\
\text { patients }\end{array}$ & $\begin{array}{l}\text { Median } \\
\text { survival }(95 \% \mathrm{CI})\end{array}$ & $P^{\mathrm{a}}$ \\
\hline \multicolumn{3}{|l|}{ Age (years) } & 0.123 \\
\hline$\leq$ Median & 51 & $5.6(3.2-8.1)$ & \\
\hline$>$ Median & 51 & $5.7(3-8.3)$ & \\
\hline \multicolumn{3}{|l|}{ Sex } & 0.272 \\
\hline Female & 45 & $5.4(3.1-7.6)$ & \\
\hline Male & 57 & $5.7(3.4-8)$ & \\
\hline \multicolumn{3}{|c|}{ Type of resection } & $<0.001$ \\
\hline No resection & 42 & $2.8(1.4-4.2)$ & \\
\hline R0 & 28 & $15.8(11-20.7)$ & \\
\hline $\mathrm{R} 1$ & 12 & $5.2(0-11.1)$ & \\
\hline $\mathrm{R} 2$ & 20 & $5.6(4.1-7.2)$ & \\
\hline \multicolumn{3}{|l|}{ pT category ${ }^{\mathrm{b}}$} & 0.019 \\
\hline pT2-pT3 & 47 & $12.9(10.4-15.4)$ & \\
\hline pT4 & 13 & $5.5(3.2-7.9)$ & \\
\hline \multicolumn{3}{|c|}{ pN category $(\text { UICC) })^{\mathrm{b}}$} & 0.799 \\
\hline pNO & 3 & $4.3(1.7-6.9)$ & \\
\hline $\mathrm{pN} 1$ & 22 & $11.5(7.8-15.2)$ & \\
\hline $\mathrm{pN} 2$ & 25 & $12.4(3.9-20.9)$ & \\
\hline pN3 & 10 & $7.5(2.4-12.6)$ & \\
\hline \multicolumn{3}{|c|}{ pN category $(\mathrm{JGCA})^{\mathrm{b}}$} & 0.447 \\
\hline pNO & 3 & $4.3(1.7-6.9)$ & \\
\hline pN1 & 13 & $16.3(13.2-19.4)$ & \\
\hline $\mathrm{pN} 2$ & 22 & $10.8(4.1-17.5)$ & \\
\hline $\mathrm{pN} 3$ & 22 & $7.4(2.7-12.3)$ & \\
\hline
\end{tabular}

CI confidence interval, UICC International Union Against Cancer, JGCA Japanese Gastric Cancer Association

${ }^{a} P$ value was computed by the Log-rank test

${ }^{\mathrm{b}} \mathrm{pT}$ and $\mathrm{pN}$ categories were reported for the 60 patients who underwent resection

3 cases), and the resection was considered potentially curative (R0) in 28 . Overall, R0 resection was achieved in $27.5 \%$ of the patients.

\section{Survival analysis}

At the follow-up end date, all the 102 patients were dead. The median (range) time of survival of the cohort was 5.7 (0.3-61) months. Seven patients died in hospital due to postoperative complications ( 3 cases) or disease progression ( 4 cases); 12 patients died within 30 days and 32 patients died within 90 days. Figure 1 depicts the time of survival for the 102 patients under study. Figure 2 shows the median time of survival according to the type of resection (no resection vs. R2 vs. R1 vs. R0). The percentages of R0 patients alive after 1,2,3, and 5 years were $68,39,14$, and $4 \%$, respectively.

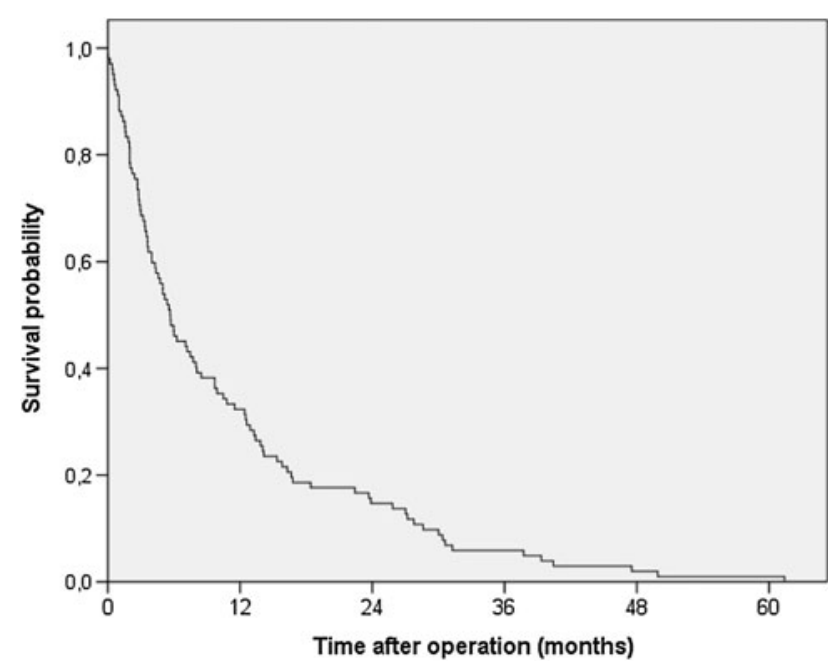

Fig. 1 Kaplan-Meier curve depicting overall survival for the 102 patients under study

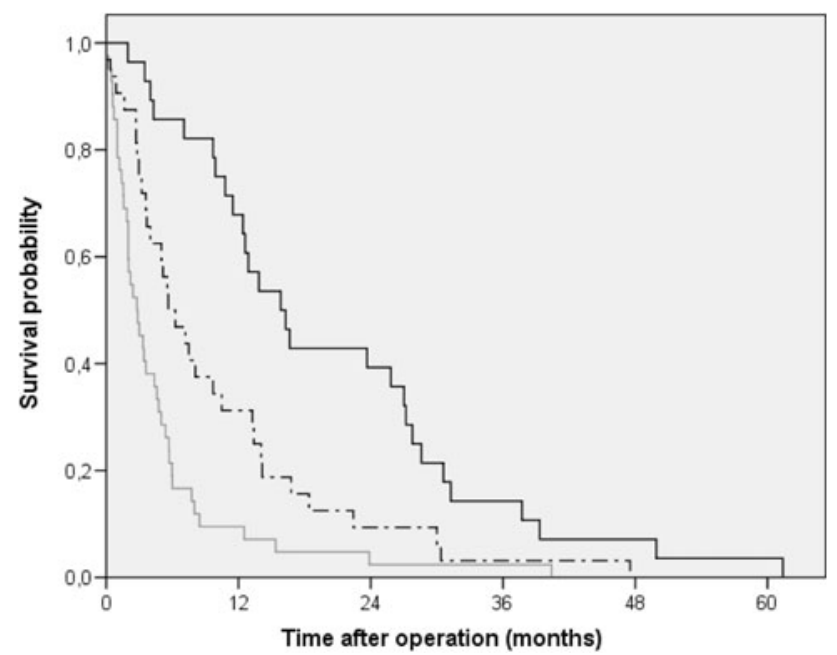

Fig. 2 Kaplan-Meier curves depicting the time of survival according to the type of resection for the 102 patients under study. Continuous black line represents potentially curative (R0) surgery, interrupted black line represents palliative (R1-2) resection, gray line represents patients in whom no resection was performed. The difference in survival probability computed by the Log-rank test was $P=0.003$ between R0 and R1-2 patients and $P=0.006$ between R1-2 and non-resected patients. The difference among the three groups was $P<0.001$

\section{Mode of recurrence}

The mode of recurrence was recorded in 36 of the 40 patients in whom an R0 or R1 resection was achieved. In 4 patients (R0 in 3 cases) the cause of death remained unknown. The recurrence was locoregional in 8 patients (R0 in 5 cases), peritoneal in 18 (R0 in 14 cases), and locoregional and peritoneal in 5 (R0 in 3 cases), whereas a systemic component of the relapse was observed in 5 patients (R0 in 3 cases). 


\section{Discussion}

The main findings of this study were: (1) patients with GLP were mostly diagnosed at a very advanced stage; (2) a potentially curative (R0) resection was possible in $<30 \%$ of the patients; (3) the chance of cure was null even after R0 resection; (4) on the whole, recurrences were intra-abdominal and were primarily related to peritoneal seeding.

Linitis plastica is a subset of diffuse gastric carcinoma with a propensity towards diffuse infiltration, massive lymph node metastasis, and peritoneal seeding. It is characterized by poorly differentiated tumor cells that diffusely infiltrate the gastric wall, thus provoking reactive fibrosis [7]. The prognosis reported in the current literature remains extremely poor $[8,9]$.

Consistent with previous reports [10-12], also in our experience the diagnosis of GLP was established very late. The tumor was judged non-resectable in $41 \%$ of the patients and, when resected, it had generally infiltrated beyond the serosal layer (98\%) and had nodal involvement (95\%). The overall percentage of $\mathrm{R} 0$ resections was confirmed to be extremely low $(27.5 \%)$.

The high number of explorative laparotomies and palliative resections indicates the inadequacy of the staging work-up adopted during the period of the study. The nonuse of staging laparoscopy certainly represented the principal reason for this failure. Thus, staging laparoscopy has to be considered mandatory in the evaluation of GLP. It may have a role in confirming the diagnosis in doubtful cases, by demonstrating undiagnosed peritoneal seeding and by evaluating tumor resectability $[13,14]$. Explorative laparotomy should be avoided completely, if we consider the minimal life expectancy of these patients (median survival time 2.8 months).

Considering survival results after potentially curative (R0) gastrectomies, no patient was alive at the end date of our study. The median survival time was 16 months and the percentages of patients alive after 1, 2, 3, and 5 years were $68,39,14$, and 4\%, respectively. Despite the extent of lymph node dissection, these data confirm previous experiences of GLP treated by primary surgery [2, 10-12] and they are comparable with cases of GC with positive peritoneal cytology $[15,16]$. Like GC with positive peritoneal cytology, GLP showed an extremely high frequency of intra-abdominal (peritoneal and/or locoregional) recurrences. In our experience, a systemic component of the recurrence was observed in $<15 \%$ of the patients. In GLP, the occurrence of the relapse is usually confined to the peritoneal cavity and the low incidence of systemic metastasis seems to indicate that the control of peritoneal seeding may provide a potential benefit. As has already been demonstrated for GC with positive peritoneal cytology or limited peritoneal carcinomatosis, hyperthermic intra-peritoneal chemotherapy (HIPEC) could represent an option in GLP and should be tested in specifically designed controlled studies [17-19].

As regards pre- or perioperative chemotherapy, GLP should be considered in multimodality treatment protocols, although no definitive and specific data are available on this entity in studies published to date [20-22]. In this regard, one should consider that a real significant benefit of actual chemotherapy agents in controlling peritoneal disease from GC has not been demonstrated [21, 23].

\section{Conclusions}

GLP represents a clinical burden for the surgeon due to the extremely poor results after primary surgery. Before surgeons lay down their arms and leave the treatment to palliative chemotherapy, specifically designed multimodality treatment protocols with preoperative chemotherapy and/or HIPEC should be tested. In this setting, an adequate preoperative work-up completed by a staging laparoscopy has to be considered mandatory.

Conflict of interest None declared.

\section{References}

1. Marrelli D, Pedrazzani C, Corso G, et al. Different pathological features and prognosis in gastric cancer patients coming from high-risk and low-risk areas of Italy. Ann Surg. 2009;250:43-50.

2. Beyrouti MI, Beyrouti R, Ben Amar M, et al. Linitis plastica. Chirurgie. 2007;36:1782-6.

3. Lauren P. The two histological main types of gastric carcinoma: diffuse and so-called intestinal-type carcinoma. An attempt at a histo-clinical classification. Acta Pathol Microbiol Scand. 1965;64:31-49.

4. Japanese Gastric Cancer Association (JGCA). The new Japanese classification of gastric carcinoma. 2nd English edition. Gastric Cancer. 1998;1:1-15.

5. American Joint Committee on Cancer. AJCC cancer staging atlas. New York: Springer Science+Business Media Inc.; 2006

6. Marrelli D, De Stefano A, de Manzoni G, Morgagni P, Di Leo A, Roviello F. Prediction of recurrence after radical surgery for gastric cancer: a scoring system obtained from a prospective multicenter study. Ann Surg. 2005;241:247-55.

7. Kodera Y, Nakanishi H, Ito S, et al. Detection of disseminated cancer cells in linitis plastica-type gastric carcinoma. Jpn J Clin Oncol. 2004;34:525-31.

8. Otsuji E, Kuriu Y, Okamoto K, et al. Outcome of surgical treatment for patients with scirrhous carcinoma of the stomach. Am J Surg. 2004;188:327-32.

9. Kodera Y, Yamamura Y, Ito S, et al. Is Borrmann type IV gastric carcinoma a surgical disease? An old problem revisited with reference to the result of peritoneal washing cytology. J Surg Oncol. 2001;78:175-81.

10. Hamy A, Letessier E, Bizouarn P, et al. Study of survival and prognostic factors in patients undergoing resection for gastric linitis plastica: a review of 86 cases. Int Surg. 1999;84:337-43. 
11. Aranha GV, Georgen R. Gastric linitis plastica is not a surgical disease. Surgery. 1989;106:758-62.

12. Moreaux J, Barrat F, Msika S. Linitis plastica of stomach. Study of 102 cases surgically treated. Results of the surgical treatment. Chirurgie. 1986;112:485-92.

13. Nakagawa S, Nashimoto A, Yabusaki H. Role of staging laparoscopy with peritoneal lavage cytology in the treatment of locally advanced gastric cancer. Gastric Cancer. 2007;10:29-34.

14. de Graaf GW, Ayantunde AA, Parsons SL, Duffy JP, Welch NT. The role of staging laparoscopy in oesophagogastric cancers. Eur J Surg Oncol. 2007;33:988-92.

15. de Manzoni G, Verlato G, Di Leo A, et al. Peritoneal cytology does not increase the prognostic information provided by TNM in gastric cancer. World J Surg. 2006;30:579-84.

16. Bentrem D, Wilton A, Mazumdar M, Brennan M, Coit D. The value of peritoneal cytology as a preoperative predictor in patients with gastric carcinoma undergoing a curative resection. Ann Surg Oncol. 2005;12:347-53.

17. Yan TD, Black D, Sugarbaker PH, et al. A systematic review and meta-analysis of the randomized controlled trials on adjuvant intraperitoneal chemotherapy for resectable gastric cancer. Ann Surg Oncol. 2007;14:2702-13.
18. Roviello F, Marrelli D, Neri A, et al. Treatment of peritoneal carcinomatosis by cytoreductive surgery and intraperitoneal hyperthermic chemoperfusion (IHCP): postoperative outcome and risk factors for morbidity. World J Surg. 2006;30:2033-40.

19. Glehen O, Schreiber V, Cotte E, et al. Cytoreductive surgery and intraperitoneal chemohyperthermia for peritoneal carcinomatosis arising from gastric cancer. Arch Surg. 2004;139:20-6.

20. Cunningham D, Allum WH, Stenning SP, et al. Perioperative chemotherapy versus surgery alone for resectable gastroesophageal cancer. N Engl J Med. 2006;355:11-20.

21. Wu AW, Xu GW, Wang HY, Ji JF, Tang JL. Withdrawn: neoadjuvant chemotherapy versus none for resectable gastric cancer. Cochrane Database Syst Rev. 2007;4:CD005047.

22. Okabe H, Ueda S, Obama K, Hosogi H, Sakai Y. Induction chemotherapy with S-1 plus cisplatin followed by surgery for treatment of gastric cancer with peritoneal dissemination. Ann Surg Oncol. 2009;16:3227-36.

23. Brigand C, Arvieux C, Gilly FN, Glehen O. Treatment of peritoneal carcinomatosis in gastric cancers. Dig Dis. 2004;22: 366-73. 